\title{
A Decomposition of the Descent Algebra of a Finite Coxeter Group
}

\section{F. BERGERON}

Dépt. de Mathematique et Informatique, Université du Quebéc à Montréal C.P. 8888, Succ. A, Montréal H3C 3P8 Canada

N. BERGERON

Department of Mathematics, Princeton, NJ 08544-1000

R.B. HOWLETT AND D.E. TAYLOR

Department of Pure Mathematics, University of Sydney, New South Wales 2006, Australia

Received May 23, 1991, Revised October 1, 1991

\begin{abstract}
The purpose of this paper is twofold. First we aim to unify previous work by the first two authors, A. Garsia, and C. Reutenauer (see [2], [3], [4], [5] and [10]) on the structure of the descent algebras of the Coxeter groups of type $A_{n}$ and $B_{n}$. But we shall also extend these results to the descent algebra of an arbitrary finite Coxeter group $W$. The descent algebra, introduced by Solomon in [14], is a subalgebra of the group algebra of $W$. It is closely related to the subring of the Burnside ring $B(W)$ spanned by the permutation representations $W / W_{J}$, where the $W_{J}$ are the parabolic subgroups of $W$. Specifically, our purpose is to lift a basis of primitive idempotents of the parabolic Burnside algebra to a basis of idempotents of the descent algebra.
\end{abstract}

Keywords: Coxeter groups, idempotents, descent algebra.

\section{Introduction}

Let $(W, S)$ be a finite Coxeter system. That is, $W$ is a finite group generated by a set $S$ subject to the defining relations

$$
(s r)^{m_{o r}}=1 \text { for all } s, r \in S
$$

where $m_{s r}$ are positive integers and $m_{s s}=1$ for all $s \in S$.

As is well known, $W$ is faithfully represented in the orthogonal group of an inner product space $V$, which has a basis $\Pi=\left\{\alpha_{s} \mid s \in S\right\}$ in bijective correspondence with $S$. The inner product is given by

$$
\left(\alpha_{s}, \alpha_{r}\right)=-\cos \left(\pi / m_{s r}\right)
$$

and the action of $W$ by

$$
s(v)=v-2\left(\alpha_{s}, v\right) \alpha_{s}
$$

for all $r, s \in S$ and $v \in V$. Thus $s$ acts as the reflection in the hyperplane orthogonal to $\alpha_{s}$, and, consequently $V$ is called the reflection representation of $W$. 
One easily checks that for all $s, r \in S$ we have $\alpha_{r}= \pm w\left(\alpha_{s}\right)$ in $V$ if and only if $r=w s w^{-1}$ in $W$.

We call the set $\Phi=\{w(\alpha) \mid w \in W, \alpha \in \Pi\}$ the root system of $W$, and $\Pi$ the set of fundamental roots. It is well known (see [7]) that $\Phi$ can be decomposed as $\Phi=\Phi^{+} \uplus \Phi^{-}$, where every element of $\Phi^{+}$(resp. $\Phi^{-}$) is a linear combination of fundamental roots with coefficients all nonnegative (resp. all nonpositive). Moreover, if $w \in W$ and $\ell(w)$ denotes the length of a minimal expression for $w$ in terms of elements of $S$, then $\ell(w)$ equals the cardinality of the set $N(w)$, where

$$
N(w)=\left\{\alpha \in \Phi^{+} \mid w(\alpha) \in \Phi^{-}\right\}
$$

Note that $\ell(v w)=\ell(v)+\ell(w)$ if and only if $N(v w)=w^{-1}(N(v)) \uplus N(w)$.

For each $J \subseteq \Pi$, the standard parabolic subgroup $W_{J}$ is the subgroup of $W$ generated by

$$
S_{J}=\left\{s \in S \mid \alpha_{s} \in J\right\}
$$

Then $\left(W_{J}, S_{J}\right)$ is also a Coxeter system. If $V_{J}$ is the subspace of $V$ spanned by $J$, then the $W$-action on $V$ yields a $W_{J}$-action on $V_{J}$, which can be identified with the reflection representation of $W_{J}$. The root system of $W_{J}$ is $\Phi_{J}=\Phi \cap V_{J}$; and we write $\Phi_{J}^{+}$for $\Phi^{+} \cap V_{J}$ for $\Phi_{J}^{-}$and $\Phi^{-} \cap V_{J}$. It is easily shown that $N(w) \subseteq \Phi_{J}^{+}$ if and only if $w \in W_{J}$.

In this paper we study the descent algebra (or Solomon algebra) $\sum(W)$ of a Coxeter group $W$. If $w \in W$, then the descent set of $w$ is defined to be

$$
D(w)=N(w) \cap \Pi=\left\{\alpha \in \Pi \mid w(\alpha) \in \Phi^{-}\right\}
$$

In terms of the generating set $S$ this corresponds to $\{s \in S \mid \ell(w s)<\ell(w)\}$. If $J \subseteq \Pi$, let

$$
X_{J}=\{w \in W \mid D(w) \cap J=\emptyset\}=\left\{w \in W \mid w(J) \subseteq \Phi^{+}\right\}
$$

and let

$$
x_{J}=\sum_{w \in X_{J}} w
$$

Define $\sum(W)$ to be the subspace of $\mathbf{Q}(W)$ spanned by all such elements $x_{J}$ (which are clearly linearly independent).

It has been shown by Solomon [14] that $\Sigma(W)$ is a subalgebra of $\mathbf{Q}(W)$. More precisely, Solomon has shown that

$$
x_{J} x_{K}=\sum_{L \subseteq K} a_{J K L} x_{L},
$$

where

$$
a_{J K L}=\left|\left\{w \in X_{J}^{-1} \cap X_{K} \mid w^{-1}(J) \cap K=L\right\}\right| .
$$

In Section 2 we shall prove these facts using techniques that will be developed further in later sections. It is easily shown (Solomon [14]) that the $x_{K}$ 's are linearly independent; thus they form a basis of $\Sigma(W)$. 
In [10] A. Garsia and C. Reutenauer have given a decomposition of the multiplicative structure of the descent algebra of the symmetric group (the Coxeter group of type $A_{n}$ ). This decomposition exploits the action of the symmetric group on the free Lie algebra in a manner reminiscent of the Poincaré-Birkhoff-Witt theorem. In [2] and [5] we showed that a similar decomposition, as well as related results, also holds for the hyperoctahedral group (type $B_{n}$ ). The object of this paper, and ongoing work, is to extend these results to the descent algebra of any finite Coxeter group.

For a general descent algebra $\sum(W)$ we shall exhibit a new basis consisting of elements $e_{k}, K \subseteq \Pi$, which are scalar multiples of idempotents, and satisfy $\sum_{K \subseteq \Pi} \mu_{K}^{\Pi} e_{K}=1$ for certain positive constants $\mu_{K}^{\Pi}$. Furthermore, for all $J, M \subseteq$ $\Pi$, when $e_{J} e_{M}$ is expressed as a linear combination of the $e_{K}$ 's, the only nonzero coefficients correspond to subsets $K$ of $M$ that are equivalent to $J$, in the sense that $J=w(K)$ for some $w \in W$. As a consequence we obtain a set of idempotents $E_{\lambda}=\sum_{k \in \lambda} \mu_{K}^{\pi} e_{K}$ indexed by equivalence classes $\lambda$ of subsets of $\Pi$, such that

$$
E_{\lambda} E_{\mu}= \begin{cases}0 & \text { if } \lambda \neq \mu \\ E_{\lambda} & \text { if } \lambda=\mu\end{cases}
$$

and $\sum_{\lambda} E_{\lambda}=1$. In fact, the $E_{\lambda}$ 's form a decomposition of the identity into primitive idempotents, and hence the right ideals of $\sum(W)$ which they generate are a full set of indecomposable projective right modules for $\sigma(W)$. Furthermore, the $E_{\lambda}$ 's induce a decomposition of the action of $\Sigma(W)$ on $\mathrm{Q}(W)$ by left multiplication:

$$
\mathbf{Q}(W)=\bigoplus_{\lambda} H_{\lambda}
$$

where $H_{\lambda}=E_{\lambda} \cdot \mathrm{Q}(W)$. We shall compute the dimension of $H_{\lambda}$ in Section 7 . Our calculations in Section 7 also show that the unique maximal submodule of $E_{\lambda} \sum(W)$ is spanned by the differences $e_{J}-e_{K}$ for $J, K \in \lambda$. This gives an alternative proof of Solomon's result that the radical of $\Sigma(W)$ is the subspace spanned all elements $x_{J}-x_{K}$ for $J$ and $K$ equivalent subsets of $\Pi$. Thus

$$
\operatorname{dim}\left(\sqrt{\sum(W)}\right)=2^{|s|}-|\Lambda|
$$

where $\Lambda$ is the set of equivalence classes of subsets of $\Pi$.

These constructions have already been carried through for all indecomposable finite Coxeter groups of type $A_{n}$ (see [10]), and of type $B_{n}$ (see [2] and [5]). Part of the study of the descent algebra has been carried through with extensive use of the computer algebra system Maple [3].

\section{The Solomon Algebra}

We start by proving some basic facts concerning the elements $x_{J}$ defined in Section 1. Proofs of results we assume can be found in Section 2.7 of Carter [7]. 
If $J \subseteq \Pi$, then each element of $W$ is uniquely expressible in the form $d u$ with $d \in X_{J}$ and $u \in W_{J}$, and here we have $\ell(d u)=\ell(d)+\ell(u)$. Thus $X_{J}$ is a set of representatives of the cosets $w W_{J}$ in $W$. Likewise, if $K \subseteq J \subseteq \Pi$, then $X_{J} \cap W_{J}$ is a set of representatives of the cosets $w W_{K}$ in $W_{J}$. In this situation we define

$$
x_{K}^{J}=\sum_{w \in W_{J} \cap X_{K}} w
$$

and note that $x_{K}^{\Pi}=x_{K}$. The next two lemmas provide analogues of induction and restriction for Solomon algebras. The connection with induction and restriction of permutation characters will be given in detail in Section 4.

LEMMA 2.1. If $K \subseteq J \subseteq \Pi$ then $X_{K}=X_{J}\left(W_{J} \cap X_{K}\right)$ and thus $x_{K}=x_{J} x_{K}^{J}$.

Proof. If $d \in X_{J}$ and $w \in W_{J} \cap X_{K}$, then $w(K) \subseteq \Phi_{J}^{+}$, whence $d w(K) \subseteq d\left(\Phi_{J}^{+}\right) \subseteq$ $\Phi^{+}$. It follows that $d w \in X_{K}$ and this shows that

$$
\left\{d w \mid d \in X_{J}, w \in W_{J} \cap X_{K}\right\} \subseteq X_{K}
$$

Since the number of products $d w$ is $\left|W: W_{J}\right|\left|W_{J}: W_{K}\right|=\left|X_{K}\right|$ we see that equality holds; and, on taking sums, we have $x_{K}=x_{J} x_{K}^{J}$.

LEMMA 2.2. For all $J, K \subseteq S$

$$
X_{K}=\biguplus_{d \in X_{J K}}\left(W_{J} \cap X_{J \cap d(K)}\right) d,
$$

where $X_{J K}=X_{J}^{-1} \cap X_{K} ;$ and thus

$$
x_{K}=\sum_{d \in X_{J K}} x_{J \cap d(K)}^{J} d
$$

Proof. First note that if $d \in X_{J K}$ and $u \in W_{J} \cap X_{J \cap d(K)}$, then $d \in X_{J}^{-1}$ and $u \in W_{J}$; so an element of $W$ can arise as a product $u d$ in at most one way.

Let $w \in X_{K}$ and write $w=u d$ with $d \in X_{J}^{-1}$ and $u \in W_{J}$. Since $\ell(u d)=$ $\ell(u)+\ell(d)$ we have $N(d) \subseteq N(u d)=N(w)$, and so $d \in X_{K}$. Thus $d \in X_{J K}$, and futhermore

$$
u(J \cap d(K)) \subseteq u d(K)=w(K) \subseteq \Phi^{+}
$$

so that $u \in W_{J \cap d(K)}$.

It remains to prove that $u d \in X_{K}$ whenever $d \in X_{J K}$ and $u \in W_{J} \cap X_{J \cap d(K)}$. Since a fundamental root cannot be nontrivially expressed as a positive linear combination of positive roots, we see that $K \cap d^{-1}\left(\Phi_{J}^{+}\right)=K \cap d^{-1}(J)$. But $d(K) \subseteq \Phi^{+}$(since $\left.d \in X_{K}\right)$ and so $d(K) \subseteq\left(\Phi^{+} \backslash \Phi_{J}^{+}\right) \cup(J \cap d(K)$ ). It follows that $u d(K) \subseteq u\left(\Phi^{+} \backslash \Phi_{J}^{+}\right) \cup u\left(J \cap d(K) \subseteq \Phi^{+}\right.$, since $u\left(\Phi^{+} \backslash \Phi_{J}^{+}\right) \subseteq \Phi^{+}$for $u \in W_{J}$, and therefore $u d \in X_{K}$, as required. 
Lemma 2.2 shows that each element of $W$ is uniquely expressible in the form $u d w$ with $w \in W_{K}, d \in X_{J K}$ and $u \in W_{J} \cap X_{J \cap d(K)}$. Moreover, in this situation $\ell(u d w)=\ell(u)+\ell(d)+\ell(w)$. It follows readily that each double coset $W_{J} w W_{K}$ contains a unique $d \in X_{J K}$, and that $W_{J} \cap d W_{K} d^{-1}=W_{J \cap(K)}$.

For $J, K \subseteq \Pi$ we write $J \sim K$ whenever $w(J)=K$ for some $w \in W$ (that is, $J$ and $K$ are equivalent) and $J \preceq K$ whenever $J$ is equivalent to a subset of $K$. The next lemma shows that this equivalence relation is the one used by Solomon in [14].

LEMMA 2.3. If $J, K \subseteq \Pi$, then $J \sim K$ if and only if $W_{J}$ and $W_{K}$ are conjugate, and $J \preceq K$ if and only if $W_{J}$ is conjugate to a subgroup of $W_{K}$.

Proof. Suppose that $w \in W$ satisfies $w^{-1} W_{J} w \subseteq W_{K}$. If $d$ is the shortest element in $W_{J} w W_{K}$, then $d^{-1} W_{J} d \subseteq W_{K}$, and therefore

$$
W_{J \cap d(K)}=W_{J} \cap d W_{K} d^{-1}=W_{J} .
$$

Thus $J \cap d(K)=J$ and therefore $d^{-1}(J) \subseteq K$. All assertions of the lemma now follow.

LEMMA 2.4. If $J \subseteq \Pi$ and $d \in W$ with $d^{-1}(J) \subseteq \Pi$, then $X_{J} d=X_{d^{-1}(J)}$.

Proof. For $w \in X_{d^{-1}(J)}$, it is clear that. $w d^{-1} \in X_{J}$, and conversely for $w \in X_{J}$, that $w d \in X_{d^{-1}(J)}$.

The following result due to Solomon is easily derived from these lemmas.

THEOREM 2.5. (Solomon) For all $J, K \subseteq \Pi$

$$
x_{J} x_{K}=\sum_{L \subseteq K} a_{J K L} x_{L}
$$

Proof.

$$
\begin{array}{rlrl}
x_{J} x_{K} & =x_{J} \sum_{d \in X_{J K}} x_{J \cap d(K)}^{J} d & & \text { by Lemma } 2.2 \\
& =\sum_{d \in X_{J K}} x_{J \cap d(K)} d & & \text { by Lemma } 2.1 \\
& =\sum_{d \in X_{J K}} x_{d^{-1}(J) \cap K} & & \text { by Lemma } 2.4 \\
& =\sum_{L} a_{J K L} x_{L} &
\end{array}
$$

Obviously $a_{J K L}=0$ when $L \nsubseteq K$. Thus the theorem is proved. 
Proposition 2.6. Let $a_{L J K}^{M}$ denote the structure constants of the descent algebra $\sum\left(W_{M}\right)$ corresponding to the $x_{N}^{M}$ basis. If $J, N \subseteq \Pi$, then

$$
x_{N} x_{J}=\sum_{K \subseteq J}\left(\sum_{L \subseteq M} a_{N M L} a_{L J K}^{M}\right) x_{K}
$$

for all $M \subseteq \Pi$ such that $J \subseteq M$. Thus the structure constants satisfy the identities

$$
a_{N J K}=\sum_{L \subseteq M} a_{N M L} a_{L J K}^{M}
$$

for all $M$ containing $J$.

Proof. We have

$$
\begin{aligned}
x_{N} x_{J} & =x_{N} x_{M} x_{J}^{M} \\
& =\left(\sum_{L \subseteq M} a_{N M L} x_{L}\right) x_{J}^{M} \\
& =\sum_{L \subseteq M} a_{N M L} x_{M} x_{L}^{M} x_{J}^{M} \\
& =\sum_{L \subseteq M} a_{N M L} x_{M}\left(\sum_{K \subseteq J} a_{L J K}^{M} x_{K}^{M}\right) \\
& =\sum_{\substack{L, K \\
L \subseteq M, K \subseteq J}} a_{N M L} a_{L J K}^{M} x_{K} .
\end{aligned}
$$

This proves the first assertion of the theorem, and comparison with

$$
x_{N} x_{J}=\sum_{K \subseteq J} a_{N J K} x_{K}
$$

completes the proof.

\section{Reduction to indecomposable finite Coxeter groups}

Throughout this section we suppose that $J$ and $K$ are mutually orthogonal subsets of $\Pi$ such that $\Pi=J \cup K$. In this case $W=W_{J} \times W_{K}$ and $\mathbf{Q} W \simeq \mathbf{Q} W_{J} \otimes \mathbf{Q} W_{K}$. We shall show that a similar decomposition holds for the Solomon algebra of $W$.

LEMMA 3.1. For $L \subseteq J$ and $M \subseteq K$ we have

$$
x_{L}^{J} x_{M}^{K}=x_{L \cup M} .
$$


Proof. Given $d \in X_{L \cup M}$ there is a unique decomposition $d=d_{J} d_{K}$ with $d_{J} \in W_{J}$ and $d_{K} \in W_{K}$. As $d_{K}$ fixes every element of $J$, it follows that

$$
d_{J}(L)=d_{J} d_{K}(L)=d(L) \subseteq \Phi^{+}
$$

and hence $d_{J} \in X_{L}^{J}$. Similarly, $d_{K} \in X_{M}^{K}$.

Conversely, if $d_{J} \in X_{L}^{J}$ and $d_{K} \in X_{M}^{K}$, then

$$
d_{J} d_{K}(L \cup M) \subseteq d_{J}\left(L \cup \Phi_{K}^{+}\right) \subseteq \Phi^{+}
$$

whence $d_{J} d_{K} \in X_{L \cup M}$. It follows that $X_{L \cup M}=X_{L}^{J} \times X_{M}^{K}$ and that $x_{L \cup M}=x_{L}^{J} x_{M}^{K}$.

As an immediate consequence of this lemma, we have

Proposition 3.2. The function $\varphi: \sum\left(W_{J}\right) \otimes \sum\left(W_{K}\right) \rightarrow \sum(W)$ defined by $\varphi(u \otimes v)=u v$, is an isomorphism of algebras.

This shows that we may reduce our discussion to the indecomposable finite Coxeter groups.

\section{The parabolic Burnside ring}

For each $J \subseteq \Pi$ we have a permutation representation of $W$ on the set $W / W_{J}$ of cosets $W_{J} w$. The orbits of $W$ on $W / W_{J} \times W / W_{K}$ have representatives of the form $\left(W_{J} d, W_{K}\right)$, where $d \in X_{J K}$; and the stablizer of $\left(W_{J} d, W_{K}\right)$ in $W$ is $d^{-1} W_{J} d \cap W_{K}=W_{d^{-1}(J) \cap K}$. Thus

$$
W / W_{J} \times W / W_{K}=\sum_{L \subseteq K} a_{J K L} W / W_{L}
$$

where the $a_{J K L}$ 's are defined as in Section 1. This proves that the representations $W / W_{J}$ span a subring $\mathcal{P B}(W)$ of the Burnside ring of $W$. We call this the parabolic Burnside ring of $W$. On comparing (3) and (1) we see that there is a homomorphism $\theta: \sum(W) \rightarrow \mathcal{P B}(W)$ which takes $x_{J}$ to the element of $\mathcal{P B}(W)$ represented by $W / W_{J}$. Note that $\theta$ is not, in general an isomorphism because $W / W_{J}$ and $W / W_{K}$ represent the same element of $\mathcal{P} \mathcal{B}(W)$ whenever $J \sim K$.

A subgroup of $W$ is said to be parabolic if it is conjugate to a standard parabolic subgroup $W_{J}$ for some $J \subseteq \Pi$. For each $v \in V$, the stabilizer in $W$ of $v$,

$$
\operatorname{Stab}_{W}(v)=\{w \in W \mid w(v)=v\}
$$

is a parabolic subgroup. Indeed, the set

$$
C=\{u \in V \mid(\alpha, u) \geq 0 \text { for all } \alpha \in \Pi\}
$$


is a fundamental domain for the action of $\mathrm{W}$, and we may choose $t \in W$ such that $t(v) \in C$. Then (see Steinberg [15])

$$
t \operatorname{Stab}_{w}(v) t^{-1}=\operatorname{Stab}_{w}(t(v))=W_{J}
$$

where $J=\{\alpha \in \Pi \mid(\alpha, t(v))=0\}$.

Since $W_{J}$ stabilizes $J^{\perp}$ it follows that $w \in W_{J}$ stabilizes $v \in V$ if and only if it stabilizes the orthogonal projection of $v$ in $V_{J}$. Hence $\operatorname{Stab}_{W_{J}}(v)$ is a parabolic subgroup of $W_{J}$. It follows by induction that the pointwise stabilizer, $\operatorname{Stab}_{W}(P)$, of an arbitrary subset $P$ of $V$, is a parabolic subgroup of $W$. Since $\operatorname{Stab}_{W}(P \cup Q)=\operatorname{Stab}_{W}(P) \cap \operatorname{Stab}_{W}(Q)$ we see that the intersection of two parabolic subgroups is again parabolic; this also follows from the fact, mentioned in Section 2 , that $W_{J} \cap d W_{K} d^{-1}=W_{J \cap d(K)}$ whenever $d \in X_{J K}$.

If $g$ is an arbitrary orthogonal transformation on $V$, define

and

$$
[V, g]=\{(1-g)(v) \mid v \in V\}
$$

$$
C_{v}(g)=\{v \in V \mid g(v)=v\}
$$

and let $\tau(g)=\operatorname{dim}[V, g]$. It is easily checked that $[V, g]$ is the orthogonal complement of $C_{v}(g)$ in $V$. Furthermore, if $0 \neq v \in V$ and $r$ is the reflection in the hyperplane orthogonal to $v$, then

$$
\tau(r g)= \begin{cases}\tau(g)+1 & \text { if } v \notin[V, g] \\ \tau(g)-1 & \text { if } v \in[V, g]\end{cases}
$$

Thus $\tau(g)$ is the length of a minimal expression for $g$ as a product of reflections. In [7] Carter proves that every element $w \in W$ can be written as a product of $\tau(w)$ reflections in $W$. (We include a proof in Lemma 4.3.)

Following Solomon [14], for $w \in W$, we define

$$
A(w)=\{y \in W \mid[V, y] \subseteq[V, w]\}=\left\{y \in W \mid C_{V}(w) \subseteq C_{V}(y)\right\} .
$$

Equivalently, $A(w)=\operatorname{Stab}_{W}\left(C_{V}(w)\right)$. In particular, $A(w)$ is a parabolic subgroup of $W$. We say that $w$ is of type $J$ if $A(w)$ is conjugate to $W_{J}$. We shall sometimes say that $w$ is of type $\lambda$, where $\lambda$ is the equivalence class of $J$, since (by Lemma 2.3) $J$ is determined by $w$ only to within equivalence. It is clear that $A\left(t w t^{-1}\right)=t A(w) t^{-1}$, and hence conjugate elements have the same type.

Observe that the maps $P \mapsto \operatorname{Stab}_{W}(P)$ and $H \mapsto C_{V}(H)$, where $H$ is a subgroup of $W$, form a Galois connection between the partially ordered set of subspaces of $V$ and the partially ordered set of subgroups of $W$, in the sense that $P \subseteq C_{V}(H)$ if and only if $H \subseteq \operatorname{Stab}_{W}(P)$. The parabolic subgroups are the closed subgroups of $W$ for this Galois connection; that is, $H$ is parabolic if and only if $H=\operatorname{Stab}_{W}\left(C_{V}(H)\right)$. Thus if $H$ is any subgroup of $W$, then $\operatorname{Stab}_{W}\left(C_{V}(H)\right)$ 
is the smallest parabolic subgroup of $W$ containing $H$. In particular, if $w \in W$, then $A(w)$ is the smallest parabolic subgroup containing $w$, and so $w$ is of type $J$ if and only if $J \subseteq \Pi$ is minimal subject to $W_{J}$ containing a conjugate of $w$.

LEMMA 4.3. Let $J \subseteq \Pi$ and suppose that $w \in W$ is of type $J$. Then

(1) if $K \subseteq \Pi$ and $W_{K}$ contains a conjugate of $w$, then $J \preceq K$,

(2) $\tau(w)=|J|$

(3) $w$ can be written as a product of $|J|$ reflections in $W$.

Proof. Replacing $w$ by a conjugate of itself, we may assume that $w \in W_{J}$. Since $w$ has type $J$ it is not contained in any proper parabolic subgroup of $W_{J}$.

If $t \in W$ and $t^{-1} w t \in W_{K}$, then $w \in W_{J} \cap t W_{K} t^{-1}$, a parabolic subgroup of $W_{J}$. It follows that $W_{J} \cap t W_{K} t^{-1}=W_{J}$. Now Lemma 3 gives $J \preceq K$, proving (1).

The generators of $W_{J}$ all fix $J^{\perp}$ pointwise, and so $J^{\perp} \subseteq C_{V}(w)$. Taking orthogonal complements gives $[V, w] \subseteq V_{J}$. If $[V, w] \neq V_{J}$, we deduce that $V_{J}$ contains a nonzero $v \in C_{V}(w)$, and hence that $w \in \operatorname{Stab}_{W_{J}}(v)$, a proper parabolic subgroup of $W_{J}$. This is a contradiction, and therefore $[V, w]=V_{J}$. Thus

$$
\tau(w)=\operatorname{dim}[V, w]=\operatorname{dim} V_{J}=|J|
$$

proving (2).

Since $[V, w]=V_{J}$ it follows from (4) that $\tau(s w)=\tau(w)-1$ whenever $s \in S_{J}$. Hence $s w$ has type $K$ for some $K \subseteq \Pi$ with $|K|=|J|-1$. Arguing by induction we deduce that $s w$ is a product of $|J|-1$ reflections in $W$, and therefore $w=s(s w)$ is a product of $|J|$ reflections.

For $J \subseteq S$, let $c_{J}$ be the product of the reflections $s, s \in S_{J}$, taken in some fixed order. The conjugacy class of $c_{J}$ in $W_{J}$ is independent of the order, and the elements of this class are called the Coxeter elements of $W_{J}$. Since $J$ is a linearly independent set it is clear that $\left[V, c_{J}\right]=V_{J}$, and so $c_{J}$ has type $J$. We note as a consequence that the parabolic subgroups of $W$ are precisely the subgroups $A(w)$.

PROPOSITION 4.4. If $J, K \subseteq \Pi$, then $c_{J}$ is conjugate to $c_{K}$ if and only if $J \sim K$.

Proof. If $c_{J}$ and $c_{K}$ are conjugate, then they are of the same type-that is, $J \sim K$. Conversely, if $J=d(K)$ for some $d \in W$, then $d S_{J} d^{-1}=S_{K}$, and so $d c_{J} d^{-1}$, being a product of the reflections in $S_{K}$, is conjugate to $c_{K}$.

Let $\varphi_{J}=\operatorname{Ind}_{W_{J}}^{W} 1$, the character of $W$ induced from the trivial character of $W_{J}$. In other words, $\varphi_{J}$ is the character corresponding to the permutation representation $W / W_{J}$. 
THEOREM 4.5. The assignment $W / W_{J} \mapsto \varphi_{J}$ defines an isomorphism $\theta$ from $\mathcal{P B}(W)$ to the ring of Q-linear combinations of the $\varphi_{J}$. Thus we may identify $\mathcal{P B}(W)$ with this ring of class functions.

Proof. If $J \sim K$, the representations $W / W_{J}$ and $W / W_{K}$ are equal in $\mathcal{P B}(W)$ and hence

$$
\varphi_{J}=\theta\left(W / W_{J}\right)=\theta\left(W / W_{K}\right)=\varphi_{K}
$$

This makes it legitimate to write $\varphi_{\lambda}$ instead of $\varphi_{J}$ where $\lambda$ is the equivalence class of $J$. For each equivalence class $\mu$ choose an element $c_{\mu}$ of type $\mu$ : for example, a Coxeter element. Since $W_{J}$ contains an element of type $K$ if and only if $K \preceq J$ it is clear that $\varphi_{\lambda}\left(c_{\mu}\right) \neq 0$ if and only if $\mu \preceq \lambda$. For a suitable ordering of the rows and columns, the matrix $\left.\varphi_{\lambda}\left(c_{\mu}\right)\right)_{\lambda, \mu}$ is upper triangular with nonzero diagonal entries. Therefore the $\varphi_{\lambda}$ are linearly independent.

Induction and restriction of characters give rise to maps between $\mathcal{P} \mathcal{B}\left(W_{J}\right)$ and $\mathcal{P B}(W)$. For the case of induction, the permutation representation $W_{J} / W_{K}$ in $\mathcal{P} \mathcal{B}\left(W_{J}\right)$ induced to $\mathcal{P B}(W)$ is simply $W / W_{K}$. By Lemma 2.1 the analogue of induction for the Solomon algebras is left multiplication by $x_{J}$. More generally, if $J \subseteq M$, define $\operatorname{Ind}_{J}^{M}: \Sigma\left(W_{J}\right) \rightarrow \sum\left(W_{M}\right)$ by

$$
\operatorname{Ind}_{J}^{M}(x)=x_{J}^{M} x
$$

The restriction of $W_{M} / W_{K}$ to $\mathcal{P} \mathcal{B}\left(W_{J}\right)$ is obtained by considering the orbits of $W_{J}$ on the cosets $W_{K} d$ in $W_{M}$. Thus

$$
\operatorname{Res}_{W_{J}}\left(W_{M} / W_{K}\right)=\sum_{d \in W_{M} \cap X_{K J}} W_{J} / W_{J \cap d^{-1}(K)}
$$

In view of this formula and Lemma 2.2 we define $\operatorname{Res}_{J}^{M}: \sum\left(W_{M}\right) \rightarrow \sum\left(W_{J}\right)$ by

$$
\operatorname{Res}_{J}^{M}\left(x_{K}^{M}\right)=\sum_{d \in W_{M} \cap X_{K J}} x_{J \cap d^{-1}(K)}^{J}=\sum_{d \in W_{M} \cap X_{J K}} x_{J \cap d(K)}^{J}
$$

The following proposition is immediate from this discussion.

Proposition 4.6. Given $J \subseteq M \subseteq \Pi$, let $\theta_{J}$, and $\theta_{M}$ be the canonical homomorphisms from $\sum\left(W_{J}\right)$ and $\sum\left(W_{M}\right)$ to $\mathcal{P} \mathcal{B}\left(W_{J}\right)$ and $\mathcal{P} \mathcal{B}\left(W_{M}\right)$ respectively. Then

and

$$
\theta_{M} \cdot \operatorname{Ind} d_{J}^{M}=\operatorname{Ind} d_{W_{J}}^{W_{M}} \cdot \theta_{J}
$$

$$
\theta_{J} \cdot \operatorname{Res}_{J}^{M}=\operatorname{Res}_{W_{J}} \cdot \theta_{M}
$$

In this context we see that Theorem 2.5 is the Solomon algebra analogue of the Mackey formula for the product of induced characters (Solomon [14]). 


\section{Dihedral groups}

In this section we give a complete description of the descent algebra of Coxeter group of type $I_{2}(p)$. That is, we take $W$ to be dihedral group with generating set $S=\{r, s\}$ and relations

$$
r^{2}=s^{2}=(s r)^{p}=1
$$

The descent algebra of $W$ has dimension 4 and it has a basis $x_{\emptyset}, x_{r}, x_{s}, x_{\Pi}$, where $\Pi=\left\{\alpha_{r}, \alpha_{s}\right\}$ is the set of fundamental roots of $W$ and where $x_{r}$ (resp. $x_{s}$ ) denotes $x_{\left\{\alpha_{r}\right\}}$ (resp. $x_{\left\{\alpha_{s}\right\}}$ ). More explicitly, we have

$$
\begin{aligned}
x_{\Pi} & =1 \\
x_{r} & =1+s+r s+s r s+r s r s+\cdots \\
x_{s} & =1+r+s r+r s r+s r s r+\cdots \\
x_{\emptyset} & =\sum_{w} w
\end{aligned}
$$

The summation for $x_{r}$ (resp. $x_{s}$ ) is over the set of all $w \in W$ with only one reduced expression, this unique expression must also end in $r$ (resp. $s$ ). The multiplication table for $\sum(W)$ is easy to compute explicitly in this case. When $p$ is even it is

\begin{tabular}{|l|l|l|l|l|}
\hline & 1 & $x_{r}$ & $x_{s}$ & $x_{\emptyset}$ \\
\hline 1 & 1 & $x_{r}$ & $x_{s}$ & $x_{\emptyset}$ \\
\hline$x_{r}$ & $x_{r}$ & $2 x_{r}+\frac{p-2}{2} x_{\emptyset}$ & $\frac{p}{2} x_{\emptyset}$ & $p x_{\emptyset}$ \\
\hline$x_{s}$ & $x_{s}$ & $\frac{p}{2} x_{\emptyset}$ & $2 x_{s}+\frac{p-2}{2} x_{\emptyset}$ & $p x_{\emptyset}$ \\
\hline$x_{\emptyset}$ & $x_{\emptyset}$ & $p x_{\emptyset}$ & $p x_{\emptyset}$ & $2 p x_{\emptyset}$ \\
\hline
\end{tabular}

whereas for $p$ odd it is

\begin{tabular}{|l|l|l|l|l|}
\hline & 1 & $x_{r}$ & $x_{s}$ & $x_{\emptyset}$ \\
\hline 1 & 1 & $x_{r}$ & $x_{s}$ & $x_{\emptyset}$ \\
\hline$x_{r}$ & $x_{r}$ & $x_{r}+\frac{p-1}{2} x_{\emptyset}$ & $x_{s}+\frac{p-1}{2} x_{\emptyset}$ & $p x_{\emptyset}$ \\
\hline$x_{s}$ & $x_{s}$ & $x_{r}+\frac{p-1}{2} x_{\emptyset}$ & $x_{s}+\frac{p-1}{2} x_{\emptyset}$ & $p x_{\emptyset}$ \\
\hline$x_{\emptyset}$ & $x_{\emptyset}$ & $p x_{\emptyset}$ & $p x_{\emptyset}$ & $2 p x_{\emptyset}$ \\
\hline
\end{tabular}


Using these tables, one can verify that for $p$ even

$$
\begin{aligned}
e_{\Pi} & =1-1 / 2 x_{r}-1 / 2 x_{s}+\frac{p-1}{2 p} x_{\emptyset} \\
e_{r} & =1 / 2\left(x_{r}-1 / 2 x_{\emptyset}\right) \\
e_{s} & =1 / 2\left(x_{s}-1 / 2 x_{\emptyset}\right) \\
e_{\emptyset} & =\frac{1}{2 p} x_{\emptyset}
\end{aligned}
$$

are mutually orthogonal idempotents whose sum is 1 . In this case the algebra $\sum(W)$ is semisimple and each equivalence class of subsets of $\Pi$ is just a singleton. Thus the idempotents $E_{\lambda}$ referred to in (2) can be identified with the idempotents listed above.

When $p$ is odd, we obtain idempotents

$$
\begin{aligned}
e_{\Pi} & =1-1 / 2 x_{r}-1 / 2 x_{s}+\frac{p-1}{2 p} x_{\emptyset} \\
e_{\tau} & =x_{r}-1 / 2 x_{\emptyset} \\
e_{s} & =x_{s}-1 / 2 x_{\emptyset} \\
e_{\emptyset} & =\frac{1}{2 p} x_{\emptyset}
\end{aligned}
$$

In this case there are only three equivalence classes of subsets of $\Pi: \Pi,\left\{\left\{\alpha_{r}\right\}\right.$, $\left.\left\{\alpha_{s}\right\}\right\}$ and $\{\emptyset\}$. The only nonzero products between distinct $e_{K}$ 's are

$$
e_{s} e_{r}=e_{r} \text { and } e_{r} e_{s}=e_{s}
$$

Thus the $E_{\lambda}$ 's of (1.2) can be taken to be

$$
e_{\Pi}, 1 / 2\left(e_{r}+e_{s}\right) \text {, and } e_{\emptyset}
$$

The radical of $\sum(W)$ is spanned by the nilpotent element $e_{r}-e_{s}$.

In preparation for Section 7, we reconsider part of this construction in the context of a general Coxeter system $(W, S)$. For two elements $r, s \in S$ we compute the product $x_{s} x_{r}$. (Again, we abbreviate $x_{\left\{\alpha_{r}\right\}}$ to $x_{r}$ ). A direct application of (1) gives

$$
x_{s} x_{r}=\rho_{s}^{r} x_{r}+\pi_{s}^{r} x_{\emptyset}
$$

where $\rho_{s}^{r}=\left|\left\{w \mid \alpha_{s}=w\left(\alpha_{r}\right)\right\}\right|$.

Observe that for any $f=\sum_{w} f_{w} w$ in $\mathbf{Q}(W)$, we have $f x_{\emptyset}=x_{\emptyset} f=\left(\sum_{w} f_{w}\right) x_{\emptyset}$. In particular, $x_{r} x_{\emptyset}=\frac{1}{2}|W| x_{\emptyset}$ and multiplying (7) by $x_{\emptyset}$ gives

$$
1 / 2 \rho_{s}^{r}+\pi_{s}^{r}=1 / 4|W|
$$

Thus (7) becomes

$$
x_{s} x_{r}=\rho_{s}^{r}\left(x_{r}-1 / 2 x_{\emptyset}\right)+1 / 4|W| x_{\emptyset}
$$


This identity suggests that for any Coxeter group we set

$$
e_{s}=\frac{1}{\rho_{s}^{s}}\left(x_{s}-1 / 2 x_{\emptyset}\right)
$$

It then follows easily that $e_{s}$ is an idempotent, and (more generally),

$$
e_{s} e_{r}=\frac{\rho_{s}^{r}}{\rho_{s}^{s}} e_{r}
$$

Clearly, if $s$ and $r$ are not conjugate, then $\rho_{s}^{r}=0$. But if they are conjugate, then there exists $d \in W$ such that $\alpha_{s}=d\left(\alpha_{r}\right)$ and then

$$
\rho_{s}^{T}=\left|\left\{w \in W \mid \alpha_{s}=w\left(\alpha_{r}\right)\right\}\right|=\left|\operatorname{Stab}_{W}\left(\alpha_{s}\right) d\right|=\rho_{s}^{s}
$$

Note that $\operatorname{Stab}_{W}\left(\alpha_{s}\right)$ is a subgroup of index 2 in the centralizer of $s$. Thus we have

$$
e_{s} e_{r}= \begin{cases}e_{r} & \text { if } s \text { and } r \text { are conjugate } \\ 0 & \text { otherwise }\end{cases}
$$

Let $\lambda(s)$ denote the equivalence class of $\alpha_{s} \in \Pi$. From the calculations just completed we conclude the following.

PROPOSITION 5.5. In any Coxeter group, for all $s \in S$, the elements

$$
E_{\lambda_{s}}=\frac{1}{|\lambda(s)|} \sum_{\alpha_{r} \in \lambda(s)} e_{r}
$$

are idempotents, and if $s$ and $r$ are not conjugate, then

$$
E_{\lambda(s)} E_{\lambda(r)}=0
$$

We generalize this result to all equivalence classes $\lambda$ in Section 7 .

\section{Idempotents in the parabolic Burnside ring}

The Q-algebra $\mathcal{P B}(W)$ is isomorphic to an algebra of functions, and therefore it has a basis of idempotent elements. Specifically, if we define

$$
\xi_{\lambda}=\sum_{\mu} \nu_{\lambda \mu} \varphi_{\mu}
$$

where the coefficient matrix $\left(\nu_{\lambda \mu}\right)$ is the inverse of the matrix $\left(\varphi_{\lambda}\left(c_{\mu}\right)\right)$ that appears in the proof of Theorem 4.5, then

$$
\xi_{\lambda}\left(c_{\mu}\right)= \begin{cases}0 & \text { if } \lambda \neq \mu \\ 1 & \text { if } \lambda=\mu\end{cases}
$$


and it follows that $\xi_{\lambda}$ is idempotent. The next theorem shows that (9) holds when $c_{\mu}$ is an arbitrary element of type $\mu$.

THEOREM 6.2. Let $J, K \subseteq \Pi$ and let $c \in W$ be any element of type $J$. Then $\varphi_{K}(c)=a_{K J J}$, the number of $d \in X_{K J}$ such that $d(J) \subseteq K$. In particular, $a_{K J J}$ depends only on the equivalence classes of $K$ and $J$.

Proof. Without loss of generality we may suppose that $c \in W_{J}$. By Mackey's formula, the restriction of $\varphi_{K}$ to $W_{J}$ is

$$
\operatorname{Res}_{W_{J}}\left(\operatorname{Ind}_{W_{K}}^{W} 1\right)=\sum_{d \in X_{K J}} \operatorname{Ind}_{W_{d^{-1}(K) \cap J}^{W J}}^{W_{J}} 1
$$

But since $c$ is not contained in any proper parabolic subgroup of $W_{J}$, the character $\operatorname{Ind}_{W_{d^{-1}(K) n J}}^{W_{J}} 1$ vanishes on $c$ unless $d^{-1}(K) \cap J=J$, in which case it takes the value 1 .

For $J \subseteq \Pi$, let $N_{J}=\{w \in W \mid w(J)=J\}$. Then $N_{J}$ is the intersection of $X_{J}$ and the normalizer of $W_{J}$, whereas $\left|N_{J}\right|=a_{J J J}$ is the index of $W_{J}$ in its normalizer.

For convenience we define $\xi_{J}=\xi_{\lambda}$ and $\nu_{J K}=\nu_{\lambda \mu}$ whenever $J \in \lambda$ and $K \in \mu$. For $J \subseteq K \subseteq \Pi$, let $\xi_{J}^{K}$ be the primitive idempotent of $\mathcal{P B}\left(W_{K}\right)$ that takes the value 1 on elements of type $J$ relative to $W_{K}$.

The next two propositions descrive the effect of the restriction and induction maps on these idempotents.

Proposition 6.3. Let $J, K \subseteq \Pi$ and let $J_{1}, J_{2}, \ldots, J_{h}$ be representatives of the $W_{K}$-equivalence classes of subsets of $K$ that are $W$-equivalent to $J$. Then

$$
\operatorname{Res}_{W_{K}} \xi_{J}=\sum_{i=1}^{h} \xi_{J_{i}}^{K}
$$

In particular, $\operatorname{Res}_{W_{K}} \xi_{J}=0$ if $J$ is not equivalent to any subset of $K$.

Proposition 6.4. If $J \subseteq K \subseteq \Pi$, then

$$
\operatorname{Ind} d_{W_{K}}^{W} \xi_{J}^{K}=\frac{\left|N_{J}\right|}{\left|W_{K} \cap N_{J}\right|} \xi_{J}
$$

Proof. Suppose at first that $J=K$ and that $c \in W_{J}$ is an element of type $J$. Then $A(c)=W_{J}$ and therefore $x^{-1} c x \in W_{J}$ if and only if $x$ is in the normalizer of $W_{J}$. So $\left(\operatorname{Ind}_{W_{J}}^{W} \xi_{J}^{J}\right)(c)=\left|N_{J}\right|$. It is clear that $\operatorname{Ind}_{W_{J}}^{W} \xi_{J}^{J}$ vanishes everywhere except at elements of type $J$, and therefore $\operatorname{Ind}_{W_{J}}^{W} \xi_{J}^{J}=\left|N_{J}\right| \xi_{J}$. 
In general, we have

$$
\operatorname{Ind}_{W_{K}}^{W} \xi_{J}^{K}=\operatorname{Ind}_{W_{K}}^{W}\left(\frac{1}{\left|W_{K} \cap N_{J}\right|} \operatorname{Ind}_{W_{J}}^{W_{K}} \xi_{J}^{J}\right)=\frac{\left|N_{J}\right|}{\left|W_{K} \cap N_{J}\right|} \xi_{J}
$$

For the purposes of calculation, the following theorem is sometimes more useful than Theorem 6.2. The quantities $\left|N_{J}\right| /\left|W_{K} \cap N_{J}\right|$ can be obtained from the tables in Howlett [11].

THEOREM 6.5. Let $J \preceq K \subseteq \Pi$ and let $J_{1}, J_{2}, \ldots, J_{h}$ be representatives of the $W_{K}$-equivalence classes of subsets of $K$ that are $W$-equivalent to $J$. If $c \in W$ is of type $J$, then

$$
\varphi_{K}(c)=\sum_{i=1}^{h} \frac{\left|N_{J}\right|}{\left|W_{K} \cap N_{J_{i}}\right|}
$$

Proof. By definition, $\sum \xi_{L}^{K}=1$, where $L$ runs through representatives of the $W_{K}$-equivalence classes of subsets of $K$. Inducing to $W$ and using Proposition 6.4 gives

$$
\varphi_{J}=\sum_{L} \frac{\left|N_{L}\right|}{\left|W_{K} \cap N_{L}\right|} \xi_{L}
$$

Since $\xi_{L}(c)=1$ if and only if $L \sim J_{i}$ for some $i$, evaluation at $c$ completes the proof.

This theorem is also a consequence of the fact that $\left|N_{J}\right| /\left|W_{K} \cap N_{J_{i}}\right|$ is the number of $d \in X_{K J}$ such that $d(J) \subseteq K$ and $d(J)$ is $W_{K}$-equivalent to $J_{i}$.

Let $C(J)$ be the set of elements of type $J$ and note that $C(J)$ depends only on the equivalence class of $J$.

The main result of this section yields a remarkable formula for the coefficients $\nu_{J K}$ in the case $K=\emptyset$.

THEOREM 6.6. If $m_{1}, m_{2}, \ldots, m_{n}$ are the exponents of $W$, then

$$
\nu_{\Pi \emptyset}=(-1)^{n} \frac{m_{1} m_{2} \cdots m_{n}}{|W|}
$$

Proof. If $\varepsilon$ is the sign character of $W$, then by Frobenius reciprocity

$$
\left(\varphi_{J}, \varepsilon\right)= \begin{cases}1 & J=\emptyset \\ 0 & J \neq \emptyset\end{cases}
$$

and therefore $\nu_{\Pi \emptyset}=\left(\xi_{\Pi}, \varepsilon\right)$. By definition of the inner product, 


$$
\begin{aligned}
\left(\xi_{\Pi}, \varepsilon\right) & =|W|^{-1} \sum_{w \in W}(-1)^{\tau(w)} \xi_{\Pi}(w) \\
& =(-1)^{n}|C(\Pi)| /|W|
\end{aligned}
$$

A well-known formula of Shephard and Todd [12] (see also Solomon [13]) states that

$$
\sum_{\omega \in W} t^{\tau(\omega)}=\left(1+m_{1} t\right)\left(1+m_{2} t\right) \cdots\left(1+m_{n} t\right)
$$

Lemma 4.3 (2) shows that $\tau(\omega)=n$ if and only if $\omega$ is of type $\Pi$. Thus $m_{1} m_{2} \cdots m_{k}$ is the number of elements of type $\Pi$ in $W$. This completes the proof.

COROLLARY 6.7. Let $J \subset \Pi$ with $|J|=k$ and let $m_{1}, m_{2}, \ldots, m_{k}$ be the exponents of $W_{J}$. Then

$$
\nu_{J \emptyset}=(-1)^{k} \frac{m_{1} m_{2} \cdots m_{k}}{\left|N_{W}\left(W_{J}\right)\right|}
$$

where $N_{W}\left(W_{J}\right)$ is the normalizer in $W$ of $W_{J}$.

Proof. To see this, apply Theorem 6.6 to $W_{J}$ and then use Proposition 6.4.

It is also interesting to observe that

$$
|W| \sum_{\mu} \nu_{J \mu}=|C(J)|
$$

The proof is obtained by taking the inner product of $\xi_{J}=\sum \nu_{J_{\mu}} \varphi_{\mu}$ with the trivial character and using the fact that $\left(\varphi_{\mu}, 1\right)=1$ for all $\mu$.

A similar calculation, but taking the inner product of $\xi_{J}$ with the sign character of $W_{L}$ induced to $W$ gives

$$
\sum_{\mu} \nu_{J \mu} a_{L \mu \theta}=(-1)^{|J|} \frac{\left|C(J) \cap W_{L}\right|}{\left|W_{L}\right|}
$$

\section{Idempotents in the Solomon algebra}

Our main aim in this section is to construct the elements $e_{J} \in \sum(W)$ mentioned in Section 1 . These elements are mapped by $\theta: \sum(W) \rightarrow \mathcal{P B}(W)$ to scalar multiples of the idempotents $\xi_{J}$ defined in Section 6 , and are themselves scalar multiples of idempotents. Moreover, the $e_{J}$ and the analogous elements $e_{J}^{K} \in \sum\left(W_{K}\right)$ are related by analogues of Propositions 6.3 and 6.4 . 
We begin by defining certain positive constants $\mu_{K}^{J}$ for all $K \subseteq J \subseteq \Pi$. For $K \subseteq \Pi$ we choose $\mu_{K}^{\Pi}$ arbitrarily, and then we put

$$
\mu_{K}^{J}=\sum_{\substack{\omega \in X_{J} \\ \omega(K) \subseteq \Pi}} \mu_{\omega(K)}^{I}
$$

For convenience, we define $\mu_{K}^{J}=0$ if $K \nsubseteq J$.

Inverting the upper triangular matrix $\left(\mu_{K}^{J}\right)$ yields constants $\beta_{K}^{J}$ such that $\beta_{K}^{J}=0$ if $J \nsubseteq K$ and

$$
\sum_{K} \mu_{K}^{J} \beta_{L}^{K}=\delta_{J L}=\sum_{K} \beta_{L}^{K} \mu_{K}^{J}
$$

where $\delta_{J L}$ is the Kronecker delta. For $J \subseteq M \subseteq \Pi$ we define $e_{J}^{M} \in \sum\left(W_{M}\right)$ by

$$
e_{J}^{M}=\sum_{K \subseteq M} \beta_{K}^{J} x_{K}^{M}
$$

noting that the coefficient of $x_{K}^{M}$ in $e_{J}^{M}$ is zero unless $K \subseteq J$. Let $e_{J}=e_{J}^{\Pi}$.

From Lemma 2.1 and this definition we immediately derive the analogue of Proposition 6.4.

Proposition 7.3. If $K \subseteq L \subseteq M$, then $e_{K}^{M}=x_{L}^{M} e_{K}^{L}$; that is, $e_{K}^{M}=\operatorname{Ind} d_{L}^{M}\left(e_{K}^{L}\right)$.

Observe that if $K \subseteq J$ and $L \sim K$, then

$$
\left\{\omega \in X_{J} \mid \omega(K)=L\right\}=\left\{\omega \in X_{L J} \mid \omega^{-1}(L) \cap J=K\right\}
$$

and so $(10)$ can be restated as

$$
\mu_{K}^{J}=\sum_{L \sim K} \mu_{L}^{\Pi} a_{L J K}
$$

Our first lemma shows that (12) remains true when $\Pi$ is replaced by $M \subseteq \Pi$.

LEMMA 7.5. If $J, K \subseteq M \subseteq \Pi$ then

$$
\mu_{K}^{J}=\sum_{L} \mu_{L}^{M} a_{L J K}^{M}
$$

where the $a_{L J K}^{M}$ are the structure constants of $\sum\left(W_{M}\right)$, and $L$ runs through subsets of $M$ that are $W_{M}$-equivalent to $K$.

Proof.

$$
\sum_{\substack{L \subseteq M \\ L_{\widetilde{M}^{K}}}} \mu_{L}^{M} a_{L J K}^{M}=\sum_{\substack{L \subseteq M \\ L_{\widetilde{M}^{K}}}} \sum_{N \sim L} \mu_{N}^{\Pi} a_{N M L} a_{L J K}^{M}
$$




$$
=\sum_{N \sim L} \mu_{N}^{I}\left(\sum_{\substack{L \subseteq M \\ L \widetilde{M}^{K}}} a_{N M L} a_{L J K}^{M}\right)
$$

Now $a_{N M L} a_{L J K}^{M}=0$ unless $L \preceq N$ and $K$ is $W_{M}$-equivalent to a subset of $L$, and when $K \sim N$ this forces $K$ to be $W_{M}$-equivalent to $L$. Hence the condition $L_{\widetilde{M}} K$ on the inner sum is superfluous, and so Proposition 2.6 gives

$$
\begin{aligned}
\sum_{L} \mu_{L}^{M} a_{L J K}^{M} & =\sum_{N \sim K} \mu_{N}^{\Pi} a_{N J K} \\
& =\mu_{K}^{J} \quad \text { by (7.4). }
\end{aligned}
$$

Associativity in $\sum\left(W_{M}\right)$ gives the following relation on the structure constants:

$$
\sum_{J \subseteq M} a_{L J K}^{M} a_{P N J}^{M}=\sum_{Q \subseteq M} a_{L P Q}^{M} a_{Q N K}^{M}
$$

for all subsets $L, P, N, K$, of $M$. If $L$ and $K$ are $W_{M}$-equivalent then the only nonzero terms on the right-hand side come from $Q$ which are in this same $W_{M}$-equivalence class. So multiplying (13) by $\mu_{L}^{M}$ and summing over $L \subseteq M$ that are $W_{M}$-equivalent to $K$ gives (by Lemma 7.5)

$$
\begin{aligned}
\sum_{J \subseteq M} a_{P N J}^{M} \mu_{K}^{J} & =\sum_{Q_{\tilde{M}^{K}}}\left(\sum_{L_{\widetilde{M}^{Q}}} \mu_{L}^{M} a_{L P Q}^{M}\right) a_{Q N K}^{M} \\
& =\sum_{Q \sim K} \mu_{Q}^{P} a_{Q N K}^{M}
\end{aligned}
$$

for all $P, N, K \subseteq M$.

We can now prove the main facts concerning multiplication of the $e_{J}^{M}$.

THEOREM 7.8. If $N, J \subseteq M \subseteq \Pi$ then

$$
e_{J}^{M} x_{N}^{M}=\sum_{\substack{K \subseteq N \\ \kappa_{\widetilde{M}^{J}}}} a_{J N K}^{M} e_{K}^{M}
$$

Note that the coefficient $a_{J N K}^{M}$ is the number of $v \in W_{M} \cap X_{N}$ such that $v(K)=J$.

Proof. If $J, L \subseteq M$ then $\sum_{K \subseteq M} \mu_{K}^{J} \beta_{L}^{K}=\delta_{J L}$. Hence multiplying (14) through by $\beta_{L}^{K}$ and summing over $K \subseteq M$ gives

$$
a_{P N L}^{M}=\sum_{K \subseteq M} \sum_{Q_{\widetilde{M}^{K}}} \mu_{Q}^{P} a_{Q N K}^{M} \beta_{L}^{K}
$$




$$
=\sum_{Q \subseteq M} \mu_{Q}^{P}\left(\sum_{K_{\tilde{M}^{Q}}} a_{Q N K}^{M} \beta_{L}^{K}\right)
$$

Multiplying through by $\beta_{P}^{J}$, where $J \subseteq M$, and summing over $P \subseteq M$ gives

$$
\sum_{P \subseteq M} \beta_{P}^{J} a_{P N L}^{M}=\sum_{K_{\widetilde{M}^{J}}} a_{J N K}^{M} \beta_{L}^{K}
$$

Multiplying this by $x_{L}^{M}$ and summing over $L \subseteq M$ gives

$$
\sum_{P \subseteq M} \beta_{P}^{J} x_{P}^{M} x_{N}^{M}=\sum_{K_{\widetilde{M}^{J}}} a_{J N K}^{M}\left(\sum_{L \subseteq M} \beta_{L}^{K} x_{L}^{M}\right)
$$

So, by (11), $e_{J}^{M} x_{N}^{M}=\sum_{K \sim J} a_{J N K}^{M} e_{K}^{M}$, as required (since $a_{J N K}^{M}=0$ for $K \nsubseteq N$ ).

COROLlaRY 7.10. If $L, J \subseteq M \subseteq \Pi$, then

$$
e_{J}^{M} e_{L}^{M}=\sum_{\substack{K \in L \\ \tilde{K}_{\widetilde{M}^{J}}}}\left(\sum_{N} a_{J N K}^{M} \beta_{N}^{L}\right) e_{K}^{M}
$$

where the inner sum is over $N$ such that $K \subseteq N \subseteq L$.

Proof. Simply write $e_{L}^{M}=\sum_{N \subseteq L} \beta_{N}^{L} x_{N}^{M}$ and use Theorem 7.8.

Corollary 7.10 shows that for each $W_{M}$-equivalence class $\lambda$, the elements $e_{J}^{M}$, for $J \in \lambda$, span a right ideal $I(M, \lambda)$ of $\sum\left(W_{M}\right)$. Furthermore, $\sum\left(W_{M}\right)$ is the direct sum of these right ideals, since the $e_{J}^{M}$, for $J \subseteq M$, form a basis of $\sum\left(W_{M}\right)$. Our next proposition shows that $\theta_{M}: \sum\left(W_{M}\right) \rightarrow \mathcal{P B}\left(W_{M}\right)$ maps the $I(M, \lambda)$ 's to the simple components of $\mathcal{P B}\left(W_{M}\right)$.

PROPOSITION 7.11. If $J \subseteq M$ and $\lambda$ is the $W_{M}$ equivalence class containing $J$, then

$$
\theta_{M}\left(e_{J}^{M}\right)=\frac{\left|W_{M} \cap N_{J}\right|}{\mu_{J}^{J}} \xi_{\lambda}^{M}
$$

Proof. We have

$$
1=x_{M}^{M}=\sum_{J \subseteq M} \mu_{J}^{M} e_{J}^{M}=\sum_{\lambda} \sum_{J \in \lambda} \mu_{J}^{M} e_{J}^{M}
$$

where $\lambda$ runs through all $W_{M}$-equivalence classes. Hence the elements $E_{\lambda}^{M}=$ $\sum_{J \in \lambda} \mu_{J}^{M} e_{J}^{M}$ are the orthogonal idempotents corresponding to the decomposition $\sum\left(W_{M}\right)=\bigoplus_{\lambda} I(M, \lambda)$. Applying the homomorphism $\theta_{M}$ we see that 
$\sum_{\lambda} \theta_{M}\left(E_{\lambda}^{M}\right)=1$ and $\theta_{M}\left(E_{\lambda}^{M}\right) \theta_{M}\left(E_{\mu}^{M}\right)=0$ whenever $\lambda \neq \mu$. It will follow that $\theta_{M}\left(E_{\lambda}^{M}\right)$ is the primitive idempotent $\xi_{\lambda}^{M}$, provided we can prove that $\theta_{M}\left(E_{\lambda}^{M}\right)$ is nonzero on elements of type $\lambda$.

We do this first in the case $\lambda=\{M\}$. Observe that if $K \subseteq M$ and $K \neq M$ then the character $\theta_{M}\left(e_{K}^{M}\right)$ vanishes on elements of type $M$, since

$$
\theta_{M}\left(e_{K}^{M}\right)=\theta_{M}\left(\operatorname{Ind}_{K}^{M}\left(e_{K}^{K}\right)\right)=\operatorname{Ind}_{W_{K}}^{W_{M}}\left(\theta_{K}\left(e_{K}^{K}\right)\right)
$$

by Proposition 4.6. Since

$$
1_{W_{M}}=\theta_{M}(1)=\sum_{K \subseteq M} \mu_{K}^{M} \theta_{M}\left(e_{K}^{M}\right)
$$

we deduce that $\theta_{M}\left(E_{\{M\}}^{M}\right)=\mu_{M}^{M} \theta_{M}\left(e_{M}^{M}\right)$ takes the value 1 on elements of type $M$.

Now consider an arbitrary $W_{M}$-equivalence class $\lambda$. Since $\theta_{M}\left(E_{\lambda}^{M}\right)=\sum_{K \in \lambda} \mu_{K}^{M}$ $\operatorname{Ind}_{W_{K}}^{W_{M}}\left(\theta_{K}\left(e_{K}^{K}\right)\right)$, and since $\theta_{K}\left(e_{K}^{K}\right)$ takes a positive value on elements of type $K$, it follows that $\theta_{M}\left(E_{\lambda}^{M}\right)$ is nonzero on elements of type $\lambda$, as required.

Thus we have proved that $\theta_{M}\left(E_{\lambda}^{M}\right)=\xi_{\lambda}^{M}$ for all $\lambda$, and in particular, $\theta_{M}\left(e_{M}^{M}\right)=$ $\left(1 / \mu_{M}^{M}\right) \xi_{M}^{M}$. Applying this with $M$ replaced by $J$ we find that

$$
\theta_{M}\left(e_{J}^{M}\right)=\operatorname{Ind}_{W_{J}}^{W_{M}}\left(\frac{1}{\mu_{J}^{J}} \xi_{J}^{J}\right)=\frac{\left|W_{M} \cap N_{J}\right|}{\mu_{J}^{J}} \xi_{J}^{M}
$$

by Proposition 6.4 .

COROLLARY 7.12. Let $\lambda$ be a $W_{M}$-equivalence class of subsets of $M$ and let $L, J \in \lambda$. Then

$$
e_{J}^{M} e_{L}^{M}=\frac{\left|W_{M} \cap N_{J}\right|}{\mu_{J}^{J}} e_{L}^{M}
$$

Proof. The only subset $K$ of $L$ such that $K_{\widetilde{M}^{J}}$ is $K=L$, and so Corollary 7.10 shows that $e_{J}^{M} e_{L}^{M}$ is a scalar multiple of $e_{L}^{M}$. Applying $\theta_{M}$ and using Proposition 7.11 determines the scalar.

Note also that the scalar $\left|W_{M} \cap N_{J}\right| / \mu_{J}^{J}$ depends only on the $W_{M}$-equivalence class $\lambda$ containing $J$. Indeed, it is easily shown (by use of Lemma 7.5, for example) that

$$
\frac{\left|W_{M} \cap N_{J}\right|}{\mu_{J}^{J}}=\left(\sum_{N \in \lambda} \mu_{N}^{M}\right)^{-1}=a_{J L L}^{M} \beta_{L}^{L}
$$

for all $L \in \lambda$.

It is immediate from Corollary 7.12 that the right ideal $I(M, \lambda)$ is generated by an element $\sum_{J \in \lambda} \rho_{J} x_{J}^{M}$ such that $\sum_{J \in \lambda} \rho_{J} \neq 0$. In particular, $I(M, \lambda)$ is an 
indecomposable. By standard theory (see [1]), it follows that the $I(M, \lambda)$ 's form a full set of projective indecomposable modules for $\sum\left(W_{M}\right)$. Since $\theta_{M}(I(M, \lambda))$ is one-dimensional, we deduce that $I(M, \lambda) \cap \operatorname{ker} \theta_{M}$ has codimension 1 in $I(M, \lambda)$, and is the unique maximal submodule of $I(M, \lambda)$. Hence the kernel of $\theta_{M}$, being the direct sum of the subspaces $I(M, \lambda) \cap \operatorname{ker} \theta_{M}$, is the radical of $\sum\left(W_{M}\right)$.

The following proposition provides the analogue of Proposition 6.3.

Proposition 7.14. Let $J, K \subseteq M \subseteq \Pi$. Then

$$
\operatorname{Res}_{K}^{M}\left(e_{J}^{M}\right)=\sum_{\substack{Q \subseteq K \\ \widetilde{M}_{\mathbb{M}^{J}}}} a_{J K Q}^{M} e_{Q}^{K}
$$

Proof. Since $e_{J}^{M}=\sum_{L \subseteq M} \beta_{L}^{J} x_{L}^{M}$, the definition of $\operatorname{Res}_{K}^{M}$ gives

$$
\begin{aligned}
\operatorname{Res}_{K}^{M}\left(e_{J}^{M}\right) & =\sum_{L \subseteq M} \beta_{L}^{J}\left(\sum_{d \in W_{M} \cap X_{L K}} x_{d^{-1}(L) \cap K}^{K}\right) \\
& =\sum_{L \subseteq M} \sum_{N \subseteq M} \beta_{L}^{J} a_{L K N}^{M} x_{N}^{K} \\
& =\sum_{N \subseteq M} \sum_{Q_{\tilde{M}^{J}}^{J}} a_{J K Q}^{M} \beta_{N}^{Q} x_{N}^{K}
\end{aligned}
$$

by (15). By definition we have $e_{Q}^{K}=\sum_{N \subseteq M} \beta_{N}^{Q} x_{N}^{K}$, and the result follows.

As our final result we determine the dimension of the right ideal $H_{\lambda}$ of $\mathbf{Q}(W)$ generated by the idempotent $E_{\lambda}^{\Pi}$.

THEOREM 7.15. The dimension of $H_{\lambda}$ is the number of elements of $W$ of type $\lambda$.

Proof. The dimension of $H_{\lambda}$ is the trace of $E_{\lambda}^{\Pi}$ in the regular representation of $W$. This can be determined by expressing $E_{\lambda}^{\Pi}$ as a linear combination of elements of $W$ and multiplying the coefficient of 1 by $|W|$.

Now $E_{\lambda}^{\Pi}=\sum_{J \in \lambda} \mu_{J}^{\Pi} e_{J}^{\Pi}=\sum_{J \in \lambda} \sum_{K \subseteq \Pi} \mu_{J}^{\Pi} \beta_{K}^{J} x_{K}$, and since 1 occurs in each $x_{K}$ with coefficient 1 , we deduce that

$$
\operatorname{dim} H_{\lambda}=|W| \sum_{J \in \lambda} \sum_{K \subseteq \Pi} \mu_{J}^{\Pi} \beta_{K}^{J}
$$

Let $C(\lambda)$ be the set of elements of $W$ of type $\lambda$. Then

$$
|C(\lambda)|=|W|\left(\xi_{\lambda}, 1\right)
$$




$$
\begin{aligned}
& =|W|\left(\theta\left(E_{\lambda}^{I}\right), 1\right) \\
& =|W| \sum_{J \in \lambda} \sum_{K \subseteq \Pi} \mu_{J}^{\Pi} \beta_{K}^{J}\left(\theta\left(x_{K}\right), 1\right)
\end{aligned}
$$

Since $\theta\left(x_{K}\right)=\phi_{K}$ and $\left(\phi_{K}, 1\right)=1$, we see that $|C(\lambda)|=\operatorname{dim} H_{\lambda}$, as required.

\section{Acknowledgements}

The authors are indebted to A. Garsia and C. Reutenauer for their invaluable contribution during the research portion of the work presented here. They are also grateful for the help of the computer algebra system Maple without which many of the ideas of this research would not have been born (see [3]).

\section{References}

1. J.L. Alperin, Local Representation Theory, New York, Cambridge University Press, 1986.

2. F. Bergeron and N. Bergeron, "A decomposition of the descent algebra of the hyperoctahedral group I," Journal of Algebra (accepted 1990).

3. F. Bergeron and N. Bergeron, "Symbolic manipulation for the study of the descent algebra of finite Coxeter groups," Journal of Symbolic Computation (accepted 1990).

4. F. Bergeron, A. Garsia, and C. Reutenauer, "Homomorphisms between Solomon's descent algebras," submitted.

5. N. Bergeron, "A decomposition of the descent algebra of the hyperoctahedral group II," Joumal of Algebra (accepted 1990).

6. N. Bourbaki, Groupes et Algèbres de Lie, Chapitres 4, 5 et 6, Eléments de Mathématiques, New York, Masson, 1981.

7. R.W. Carter, Finite Groups of Lie Type: Conjugacy Classes and Complex Characters, New York, Wiley, 1985.

8. R.W. Carter, "Conjugacy classes in the Weyl group," Compositio Mathematics, vol. 25, 1972, pp. $1-59$.

9. A.M. Garsia, Combinatorics of the free Lie algebra and the symmetric group, Analysis, et cetera, Research papers published in honor of Jurgen Moser's 60th birthday, ed: Paul H. Rabinowitz and Eduard Zehnder, Academic Press, 1990.

10. A. Garsia and C. Reutenauer, "A decomposition of Solomon's descent algebras," Advances in Mathematics, vol. 77, no. 2, pp. 189-262, 1989.

11. R. B. Howlett, "Normalizers of parabolic subgroups of reflection groups," Journal of the London Mathematics Society, vol. 21, pp. 62-80, 1980.

12. G. C. Shepahard and J. A. Todd, "Finite unitary reflection groups," Canadian Joumal of Mathematics, vol. 6, pp. 274-304, 1954.

13. L. Solomon, "Invariants of finite reflection groups," Nagoya Mathematics, vol. 22, pp. 57-64, 1963.

14. L. Solomon, "A Mackey formula in the group ring of a Coxeter group," Journal of Algebra, vol. 41, pp. 255-264, 1976.

15. R. Steinberg, "Endomorphisms of Linear Algebraic groups," Mem. American Mathematics Society, vol. 80, 1968, pp. 1-108. 